\title{
La obesidad en la resignificación de identidades infantiles indígenas en edad escolar en México: el caso de los pueblos Mazahua y Otomí
}

\author{
Ivonne Vizcarra-Bordi*
}

Nadia Marín-Guadarrama ${ }^{* *}$

\section{Resumen}

En México, la obesidad infantil es un grave problema de salud pública relacionado con las consecuencias de la globalización y de los mercados agroalimentarios. Esta situación ha alcanzado a los infantes indígenas otomíes y mazahuas, quienes viven una constante reformulación de sus contextos étnicos, locales y familiares intrínsecamente interconectados con los procesos de globalización y que traen como consecuencia desigualdades sociales. Para saber cómo las identidades indígenas de los infantes con edad escolar se han reformulado, este artículo presenta un estudio sobre los contextos en los que los infantes viven su cotidianeidad junto con las variables de género, etnia, pobreza y cuerpo. Se empleó una metodología cualitativa junto con datos estadístico-descriptivos obtenidos de dos comunidades indígenas: Mazahua y Otomí. Se concluye que tanto el género como la pertenencia a una etnia y la situación de pobreza hacen que se discrimine a esta población infantil, y que se le estigmatice. Así, resulta incorrecto responsabilizar sólo a las madres o a los infantes mismos por las condiciones nutricionales en que viven, ya que sus decisiones alimentarias están determinadas por instituciones gubernamentales y no gubernamentales, así como nacionales e internacionales y de empresas de alimentos nacionales y globales.

Palabras claves: Obesidad. Infancia. Identidad.

\footnotetext{
* Doctorado en Antropología Social. Maestría en economía rural. Especialista en seguridad alimentaria y nutricional y estudios de género. Investigadora del Instituto de Ciencias Agropecuarias y Rurales de la Universidad Autónoma del Estado de México. Coordinadora de Investigación y Estudios Avanzados. Miembro del Sistema Nacional de Investigadores Nivel III. ** Doctora en antropología por la Universidad Estatal de Nueva York en Albany.
} 


\section{Introducción}

Tanto en antropología como en sociología, se observa una creciente preocupación por las relaciones y vínculos existentes entre el proceso de la globalización y los cambios en las formas culturales de las sociedades locales e indígenas. Entre lo que llama la atención son las nuevas expresiones culturales complejas que van adquiriendo las infancias de una localidad, las que no sólo se aprecian enmarcadas en un proceso globalizador sino en las múltiples interconexiones socioculturales que se manifiestan en su crecimiento físico e intelectual - cuerpo-mente -, estableciendo relaciones y acciones conscientes e inconscientes sobre el sentido de pertenencia (BERGER; LUCKMANN, 2006). En este sentido, la idea de la aldea global de McLuhan (MACLUHAN; POWERS, 1993), donde las culturas tienden a homogeneizarse y en ellas producir inclusivamente infancias globales y derechos universales, ha sido ampliamente rebasada (CARLI, 2009), en gran parte por el replanteamiento de la investigación social sobre las infancias a partir de perspectivas con enfoques inter y multidisciplinarios (PROUT; JAMES, 1990). La noción del proceso de globalización deja de verse como modelo único y de homogenización cultural que basa su expansión a través de un incremento sustancial del uso de nuevas tecnologías de la información y la comunicación, del consumo de estilos de vida acompañados de estéticas corporales derivadas del comercio cultural, y del mercado neoliberal alimentario tendiente a suprimir las realidades culturales de menor poder (MELÉNDEZ-TORRES; CAÑEZ DE LA FUENTE; FRÍAS JARAMILLO, 2012; URIBE, 2006). Por otra parte, se supera también el debate que considera la pertinencia de luchar por la preservación de la diversidad cultural para hacer frente al impacto de la globalización sobre las formas locales de existir, de ser y de alimentarse, a través de conservar saberes y practicas locales desde una visión humanística, costumbrista y horizontal (BOISIER, 2001). Lo cierto es que la globalización ha llegado para permanecer por un largo período y buscará uniformar la cultura [infantil] como consecuencia del consumo de culturas globales (FRIEDMAN, 1995). Pero también es cierto que en estos procesos se da un efecto heterogéneo dada las diversidades culturales locales, las que a su vez producen marcadas diferenciaciones ante el aumento de las desigualdades sociales pre-existentes (CARLI, 2009). De aquí que de algún modo entendemos que la construcción social de la infancia en un pueblo indígena (local) no puede entenderse sin reformularse constantemente sus 
contextos étnico-locales y familiares en su interconexión con los procesos globales, y en sus consecuentes desigualdades sociales.

Este estudio plantea un acercamiento cualitativo a esas construcciones sociales de la realidad donde se configuran las identidades infantiles de dos pueblos indígenas del los valles centrales de México: uno Mazahua y otro Otomí. Consideramos que aproximarse a uno de los problemas más visibles que han tenido las interconectividades entre lo local y lo global, nos permitirá de alguna manera enunciar las aristas complejas que deben ser estudiadas en las definiciones de las infancias de la actualidad. Nos referimos a la obesidad infantil ${ }^{1}$, específicamente de escolares entre 5 y 12 años de edad.

En efecto, desde la década de los 90s, la obesidad ha sido considerada como uno de los mayores problemas de salud pública, relacionada con la globalización de los mercados agroalimentarios. La obesidad es una enfermedad crónica caracterizada por un aumento patológico de grasa corporal que se asocia a un mayor riesgo para la salud. Se produce por un balance calórico positivo, ya sea por un elevado aporte calórico, una reducción del gasto energético o la combinación de ambos (BAUDRAND; ARTEAGA; MORENO, 2010). Tal ha sido el aumento de su incidencia tanto en países desarrollados como en desarrollo que por su dimensión se ha identificado como epidemia del siglo XXI (WHO, 1998). Si bien esta asignación está en debate por su contenido políticosocial (MOFFAT, 2010), lo que es un hecho es que si a temprana edad los individuos presentan obesidad, existe una probabilidad alta y directa de presentar obesidad con síndrome metabólico durante su edad adulta. Esta enfermedad es considerada un factor de riesgo para desarrollar enfermedades crónicas degenerativas como la diabetes mellitus, dislipidemias, cáncer, hipertensión arterial y otras enfermedades cardiovasculares, además que eventualmente puede desencadenar la reducción drástica de la calidad de vida en edad reproductiva y disminuir la esperanza de vida de esas personas (LOBSTEIN; BAUR; UAUY, 2004; WRIGHT, 2001).

La Organización Mundial para la Salud (WHO), calculó que en el 2010 habían en los países en desarrollo cerca de 35 millones de niños y niñas mayores de 5 años con sobrepeso y obesidad, mientras que en los países desarrollados habían 8 millones. Una relación inversa existía hace 20 años donde de los 20 millones de infantes con ese problema la mayoría vivía en países desarrollados (WHO, 2012). Lo alarmante es la velocidad en que está aumentando la incidencia de nińos y niñas con obesidad en países que aún no han superado 
los graves problemas de desnutrición infantil, y aún más paradójico es que dentro de los mismos hogares pobres coexisten ambas patologías nutricionales (GOTTHELF; ARAMAYO; ALDERETE, 2004). De hecho podríamos estar hablando de una obesidad de la pobreza (ISUNZA, 2004), pues en países latinoamericanos se ha demostrado que existe una correlación directa entre la pobreza y la obesidad infantil (PENA; BACALLAO, 2000). Se sabe que los niños y las niñas de bajos estratos socioeconómico que nacen de bajo peso, y que son alimentados (as) antes de los 5 años con comestibles procesados industrialmente (hipercalóricos) disponibles en su entorno, ricos en grasa, sal y azúcares pero pobres en aminoácidos esenciales, vitaminas, minerales y otros micronutrientes, son una de las causas de ganar peso adiposo y desarrollar carencias nutricionales (CALZADA LEÓN, 2003). Esta transición de desnutrición a obesidad se ha dado principalmente en muchas poblaciones rurales e indígenas donde persisten patrones de consumo locales, pero que rápidamente han introducido a su dieta el consumo de alimentos industrializados (POPKIN, 2001). Aunado a esto, los cambios alimentarios por lo general vienen acompañados de un descenso drástico de la actividad física, tendiente al sedentarismo como parte de la adopción de estilos de vida occidentalizados, donde los niños y las niñas pasan más tiempo frente a la televisión y a otros entretenimientos electrónicos (AMIGO, 2003).

En México, las últimas encuestas nacionales (INSP-ENSANUT, 2012) que dan cuenta de la situación alimentaria y nutricional han observado que la prevalencia de obesidad en escolares de 5 a 11 años de edad pasó de $26.9 \%$ en 1999 a $34.4 \%$ en el 2012 siendo los niños los que tuvieron mayor incidencia con respecto a las niñas. Aunque al parecer estos índices no presentaron cambios de 2006 al 2012, siguen siendo de los más elevados a nivel mundial. Desafortunadamente estas encuestas no permiten hacer distinciones entre clases sociales, ni entre el medio rural y el medio urbano, ni mucho menos por grupos étnicos. Sin embargo, muestran resultados por grandes regiones de México, destacando que en la región Centro (territorio de nuestro interés en este estudio), ha habido un aumento de obesidad en menores de 5 ańos, pasando de $8.0 \%$ en 1999 a 9.9\% en 2012. En el mismo rango de edad del período observado, también se destacan avances significativos en la disminución de la desnutrición infantil, aunque en las zonas rurales en general y en la región Sur de México (donde se concentra la mayor parte de la población indígena), la desnutrición sigue siendo una realidad. Algunas de las causas de estos cambios nutricionales 
en la población infantil tienen que ver con los procesos globales alimentarios y otros consumos culturales, pero estos por si solos no hubieran podido penetrar tan rápido sin la existencia de un acceso monetario familiar para la compra de productos provenientes de mercados transnacionales. La dependencia de un ingreso para subsistir y la falta de políticas de desarrollo de producciones agroalimentarias locales, han hecho que cada vez los hogares rurales e indígenas no sobrevivan de sus actividades agrícolas ni artesanales. Por ello han buscado estrategias de diversificación para obtener ingresos, tales como la migración masculina, el abandono de sus actividades primarias y su incorporación a los mercados laborales precarios e informales (VIZCARRA; LUTZ, 2010). Paralelamente, las políticas de desarrollo social confeccionadas para reducir la pobreza a través de transferencias monetarias directas a las madres, tales como el programa PROGRESA y Oportunidades, forman hoy en día parte de esas estrategias para obtener ingresos de manera irregular (VIZCARRA, 2012) ${ }^{2}$.

Estas realidades contrastantes han puesto en la mira a la malnutrición de la población infantil en México. No sólo por parte de los medios de comunicación, quienes en los últimos seis años, difundieron que la incidencia de obesidad infantil mexicana ha alcanzado el primero o segundo lugar en el mundo ${ }^{3}$ o de organismos internacionales como la Organización para la Cooperación y el Desarrollo Económico que recientemente declaró que, entre sus países miembros, México ocupaba el cuarto lugar en obesidad en personas de 5 a 17 ańos de edad (SASSI, 2010), sino que ha alertado a todo el aparato gubernamental para diseñar estrategias de intervención a nivel de las instituciones de salud pública (IMSS, 2010) y educativas (VILLALOBOS, 2010).

Se han reconocido socialmente dos espacios privilegiados para ir adquiriendo rasgos de identidad según su género desde temprana edad: uno es el hogar donde se legitima el sistema de creencias que estructura el orden social de la comunidad y el otro es la escuela, donde la mayoría de niños y niñas entre 5 y 12 años de edad pasan una parte del día socializando hábitos alimentarios, juegos y otros aprendizajes socioculturales con sus pares. En este trabajo consideramos que estos espacios son propicios para estudiar las interconectividades con procesos locales y globales, que contribuyen a la redefinición de las identidades de niños y niñas que presentan obesidad. Para entender estos procesos relacionales, el escrito está dividido en tres grandes apartados. El primero ofrece un recorrido teórico sobre la construcción social 
de las identidades infantiles en la interconectividad de procesos individuales, locales y globales. El segundo apartado refiere a los contextos alimentarios y nutricionales de las dos comunidades de estudio. En la última parte se presenta el análisis de las redefiniciones de identidades infantiles en ambas comunidades en dos espacios sociales señalados: el hogar y la escuela.

\section{Identidades infantiles indígenas: una reflexión teórica sobre su construcción social}

El estudio de la resignificación de las identidades de los niños y las niñas mazahuas y otomíes a partir de constructos de género, etnicidad, pobreza y de su cuerpo se encuentra contextualizado por una realidad dominada por los procesos de globalización. En específico, los contextos de pobreza, la marginación social, los problemas de salud, las deficiencias alimentarias y la falta de apoyo al campo por parte del gobierno, son los espacios en los que los niños y las niñas indígenas cotidianamente van concretando sus identidades. De aquí que la pregunta de cómo se conforman dichos procesos requiere de una reflexión teórica sistemática y que ligue claramente el estudio de las identidades infantiles con el problema de la obesidad.

Partimos de la inexistencia de un solo concepto de infancia ya que éste es una construcción social y cultural (ARIÈS, 1962) determinada por un contexto espacio-temporal en el que se encuentran los niños y las nińas viviendo cotidianamente. En este sentido, para Frones (1993) existen múltiples infancias, las cuales dependen de la intersección entre sistemas culturales, sociales y económicos, así como de los ambientes artificiales y naturales en las que los y las infantes viven. De acuerdo a Mead (2001), los nińos y las nińas son parte de una cultura única, y a partir de ésta es que debe entenderse su existencia. Sin embargo, los procesos de globalización han afectado dichas concepciones y han hecho que los estudios se complejicen. Efectivamente, la globalización es un fenómeno social que ha originado muchos y rápidos cambios en diferentes sociedades del mundo y los roles, cuyos valores e identidades de los y las infantes están condicionados a los contextos nacionales e internacionales, ya que las acciones de las instituciones condicionan las decisiones relacionadas con el cuidado de los niños y las niñas (STEPHENS, 1995).

Por su parte, Prout y James (1990) propusieron que hay elementos que deben ser considerados para realizar estudios de las infancias a partir 
de que ésta es tomada como una categoría de análisis social que requiere ser acompañada de otras categorías tales como: clase, género y raza o etnicidad; categorías relacionales que se dan entre infantes y su propia cultura. Estudiar las infancias es pensar en los niños y las niñas como sujetos sociales activos y no como actores pasivos dentro de los procesos y de las estructuras sociales. Con esta perspectiva, conviene separarse de las propuestas teóricas que quieren ver al infante desde los conceptos de racionalidad, naturalidad y universalidad, aproximación que dominó el estudio de las infancias en las ciencias sociales hasta hace algunos años (PROUT; JAMES, 1990).

Los y las infantes indígenas internalizan desde edad temprana los estigmas sociales que los y las marginan, por lo tanto es importante fijarnos en los procesos en los que aprenden y luego internalizan dichos estigmas. Se trata de los procesos por los cuales los y las infantes están reconfigurando sus identidades. Para analizar estos procesos, Berger y Luckmann (2006) establecieron los criterios que deben considerarse a partir del estudio de la vida cotidiana, comenzando por las socializaciones primarias y secundarias por las que obligatoriamente pasan los niños y las niñas. Se sabe que la vida es un proceso que parte desde el nacimiento, pero que los procesos de objetivación de subjetividades ya están construidos por la familia, la comunidad y la sociedad en su conjunto. A los niños y niñas al crecer en un orden social preconcebido, sólo les corresponde aprender de los roles institucionales e individuales, lo que no significa necesariamente que los adoptarán totalmente y sin modificaciones e inclusive en sus etapas de crecimiento pueden rechazar esas asignaciones por múltiples razones. En este sentido las socializaciones no tienen formas lineales ni atemporales sino por lo contrario son multidimensionales y multitemporales. Por ejemplo, el proceso de cierta socialización puede verse deficiente por situaciones sociales desventajosas como es el caso de las desigualdades sociales que históricamente han marginado a las comunidades indígenas; o bien porque la socialización se interrumpen abruptamente en ciertas etapas de desarrollo infantil debido a externalidades como son conflictos geopolíticos, guerras o catástrofes naturales.

De lo anterior se puede inferir que al menos cuatro categorías de análisis están presentes en la construcción social de las infancias indígenas y que de alguna manera interfieren en las formas de socialización donde los y las infantes configuran sus identidades ante procesos de cambio y globalización: el contexto (hogar, escuela, gobierno), el género (valores y roles), la etnia (legitimaciones) y el cuerpo (imagen ideal). 


\section{Contexto}

Sin duda, los procesos históricos que configuran los contextos en que los y las infantes se socializan para pertenecer al grupo social donde nació o fue trasladado(a) por alguna situación, son elementos de análisis para entender los procesos de identidad estigmatizada (BERGER; LUCKMANN, 2006). Debido a las múltiples situaciones contextuales, se ha propuesto que una forma simple de comenzar a estudiar sus identidades es conociendo cómo, por qué y quiénes los y las que cuidan, pues son los y las que influyen el medio en el que crecen los niños y las niñas (HARKNESS; SUPER, 1983). Sean instituciones sociales como la familia nuclear o extensa, sean religiosas o de beneficencia social, $\mathrm{u}$ oficiales como la escuela e inclusive programas de asistencia social familiar, las relaciones que se establecen entre cuidadores(as) e infantes juegan un papel importante en la construcción social de la infancia en el vivir día a día, es decir en la cotidianidad.

En lo que respecta a México, las perspectivas institucionales generalmente consideran a las madres indígenas como las responsables más importantes para la crianza de los niños y las niñas. Sin embargo, los gobiernos encargados de generar políticas hacia los infantes ya sea de salud, alimentación o educación también están siendo partícipes de este proceso. Tomando esta perspectiva que engloba a toda una red de cuidado, una propuesta teórica feminista para estudiar la niñez es la ética del cuidado, porque enfatiza en la responsabilidad de quienes están a cargo de los y las infantes más que en continuar con el discurso sobre sus derechos universales (COCKBURN, 2005).

\section{Género}

Ahora bien, los estudios feministas han destacado la importancia de incluir la categoría de género en la construcción social de las identidades ya que marcan las primeras diferenciaciones sociales entre los que se espera de un niño o una niña, dependiendo del contexto donde crezca y con ello del orden social establecido en ese tiempo. Así por ejemplo encontramos que entre los Ambarras, los roles de los y las infantes son asignados hasta la edad de 10 ańos (HAMILTON, 1981). En Jamaica las jefas de familia prefieren tener niñas en lugar de niños debido a que ellas tienden a cooperar más en la reproducción de su hogar (SARGENT; HARRIS, 1992). Greenhalg (1994) encuentra una 
situación diferente en las áreas rurales de China, donde padres y madres preferían tener niños debido a las políticas del Estado que permite tener sólo un hijo aún en lugares rurales donde culturalmente el trabajo se centra más en lo que aporta un varón. Vizcarra y Marín (2006) también encuentran preferencia por tener hijo varón entre los mazahuas, no sólo por su asignación de género a las actividades agrícolas, sino por el valor que ellos tienen en una sociedad patriarcal, donde se busca siempre tener el primogénito varón a quien se le deposita el poder familiar y se le heredan los bienes materiales.

En general, los roles y el valor que tienen los infantes en la comunidad de acuerdo a su género están determinados por las actividades asignadas en su vida cotidiana, y resultan una parte nodal en la construcción de las identidades de los niños y niñas de diferentes sociedades y en la reproducción de sus grupos.

\section{Etnia}

En las décadas de los 70 s y 80 s, se realizaron estudios antropológicos desde la escuela, la cultura y la personalidad que mostraron la diversidad de formas de criar y de pensarse niño o niña. Por su parte, Wallace (1983) propone que las culturas alrededor del mundo tienen diferentes comportamientos, los cuales no obedecen a la lógica de Occidente. Es aquí donde nuestra atención se centra en los grupos étnicos que atribuyen diferentes valores y roles a los niños y a las niñas, los cuales están condicionados por un aparato ideológico propio de la comunidad, pero que a su vez también se ven influenciados por contextos más amplios con los que se relacionan diversos miembros del hogar y de la comunidad.

Cuando los discursos de la psicología de occidente relacionados con el desarrollo infantil estaban siendo incorporados en otros países sin importar que se hablaba de contextos culturales diferentes, los estudios antropológicos comenzaron a mostrar formas diferentes de crianza y de desarrollo de los infantes. Tal fue el estudio de Hamilton (1981) sobre los Ambarras, quien argumentaba que el trato a los infantes era muy indulgente, y que los castigos, los golpes o la coerción de padres y madres ocurría muy esporádicamente, dando como resultado un(a) infante con una personalidad llena de seguridad. De acuerdo a esta autora, el contacto con el mundo simbólico que los y las infantes seguían al haber crecido no presentaba sentimientos de ansiedad, de pánico u oposición de algún tipo. Como consecuencia, la transición de la 
infancia a la adultez era tranquila, sin las complicaciones de la adolescencia de Occidente. Éste y otros estudios que mostraban otras formas de vida diferentes a la occidental (véase GOTTLIEB, 2004), pronto se vieron rebasados ante la expansión de procesos globales que movilizaron no sólo bienes de consumo, sino ideas, personas, símbolos y capitales. Ello no quiere decir que todos los estudios sobre infancias en grupos étnicos se hayan centrado en diferenciar las formas de crianza particular con respecto a la cultura occidental. Tal es el caso de algunas comunidades indígenas en México, donde es recurrente el uso del castigo y el golpe para educar al infante. Pero eso no significa que dicho comportamiento sea una característica de crianza de la cultura indígena, pues los problemas de alcoholismo de los y las cuidadores(as), la situación de pobreza y marginación en la que viven los pueblos indígenas y el sistema de creencias aunado a las costumbres adoptadas después de las colonizaciones de estas poblaciones, muestran que los comportamientos violentos hacia los y las infantes provenían también de las sociedades occidentales colonizadoras (VIZCARRA; MARÍN, 2006).

\section{Cuerpo}

Existen pocos estudios sobre la construcción social de los cuerpos infantiles, y mucho menos los hay sobre los cuerpos de infantes indígenas. Aún así consideramos que el cuerpo, visto como el espacio donde se significan los aciertos y las negaciones de los ideales de una cultura, es también un "indicador inevitable de la identidad individual, del grupo de origen y de la función social encomendada, de las desigualdades existentes, y del tipo de relación que, en un contexto dado, se mantiene con el mundo" (CARBALLO; CRESPO, 2003, p. 241). De esta manera, la construcción social de los y las infantes indígenas se estructura a través de las categorías antes expuestas, las que a su vez, están siendo reestructuradas debido a las presiones de las nuevas configuraciones sociales (locales, regionales y mundiales), pero al agregar además la categoría "cuerpo", no sólo se acentúan percepciones individuales, sino que también se amplía el entramado social del pertenecer a un grupo de edad, donde se entretejen otros procesos globales que interfieren con la vida de un niño o una niña. Entre estos procesos globales, la alimentación resulta ser la que más se relaciona directamente con el cuerpo, seguida por la imagen corporal idealizada. Sin duda, sus valoraciones culturales promueven 
prácticas de aceptación o rechazo, trayendo como consecuencias graves problemas relacionados con estigmas muchas veces ajenos a las culturas locales (URIBE, 2006).

\section{Las comunidades de estudio: modos de vida, alimentación y nutrición}

En el Estado de México, ubicado en el centro del país, aún existen cinco pueblos originarios hablantes de las lenguas indígenas náhuatl, mazahua, otomí, matlazinca y tlahuica. De acuerdo con datos del Censo de Población y Vivienda de 2010 (CONAPO-INEGI, 2011), 222,394 habitantes corresponden a estos pueblos originarios. Es decir, el $1.5 \%$ de la población total de la entidad, siendo el pueblo mazahua el más numeroso al contar con 116,240 (53.85\%) hablantes de esa lengua y en segundo lugar el pueblo otomí con 97,820 (43.20\%). Por su cercanía a las capital del Estado de México y a la capital del país, su población ha establecido una relación estrecha con las grandes urbes, ya sea porque les han ofrecido fuentes de trabajo extra-agrícola, porque han emigrado a ellas, o bien porque las ciudades las han atrapado en su proceso de crecimiento urbano como lo es para varias comunidades otomíes del valle de Toluca. En ambos pueblos prevalecen condiciones de marginación y pobreza, y en la última década existe una tendencia a la feminización de la agricultura familiar (VIZCARRA, 2013), razones por las cuales los programas gubernamentales de ayuda alimentaria y combate a la pobreza las han convertido en sus "beneficiarias" más importantes (CARMONA; VIZCARRA, 2009; CEBALLOS, 2012a). Además de estas características, sus enigmas culturales y étnicos nos han atraído para realizar diferentes estudios antropológicos y nutricionales en los últimos 20 años, siendo los dos más recientes: "El maíz mesoamericano y sus escenarios de desarrollo local”", en la comunidad mazahua de San Jerónimo Mavatí (2012-), e "Inseguridades alimentaria y nutricional en comunidades periurbanas del valle de Toluca" en la comunidad de San Andrés Cuexcontitlán (2011-2012)5. En las dos investigaciones realizamos un diagnóstico comunitario etnográfico y un estudio sobre el estado nutricional (de 4 a 16 años de edad). Igualmente realizamos 40 entrevistas a las madres (10 mazahuas y 30 otomíes) de los y las infantes que presentaron obesidad para conocer la percepción del estado nutricional de sus hijos e hijas, así como de ellas mismas ${ }^{6}$. 


\section{Mazahuas de San Jerónimo Mavatí}

La comunidad pertenece al municipio de San Felipe del Progreso en el Estado de México. En el 2010 se censaron 905 habitantes (52\% mujeres), de los cuales el $25 \%$ tenían entre 5 y 12 años de edad y vivían distribuidos en 181 hogares, $15 \%$ de ellos jefaturados por mujeres. En promedio los hogares cuentan con 5 miembros. Teniendo como característica el envejecimiento de la población, ya que el $40 \%$ es mayor de 55 años de edad. Es una comunidad eminentemente rural e indígena, donde la mayoría proviene de ancestros mazahuas aunque en la actualidad sólo el $44 \%$ de la población habla el idioma. Cuenta con una escuela de pre-escolar y una escuela primaria pero no acostumbran enviar a los y las menores de 5 años a la escuela, porque se piensa que aún no están aptos y aptas para ello. Poco a poco, las madres jóvenes están rompiendo con esta creencia, adoptando comportamientos propios de los estilos de vida urbana y occidental. A pesar de que los hogares han tenido que ir modificando sus estrategias de reproducción social, esto no ha puesto en riesgo algunas estructuras fundadas en la ideología patriarcal ya que las mujeres son las que principalmente se encargan del hogar. Además, mantienen su cohesión social dentro de la comunidad a través de su participación activa en festividades cívico-religiosas propias de la cultura local o en otras costumbres mazahuas como el trabajo colectivo para mejorar la infraestructura de la comunidad o de ayuda en los procesos de producción agrícolas. Y al igual que millones de hogares rurales en México, han tenido que diversificar sus actividades económicas ya que la producción agrícola de subsistencia, principalmente de maíz nativo producido en condiciones de temporal (a una altitud de 3040 m.s.n.m.) no cubre las necesidades de alimentación. Por eso, desde la década de los ochentas la mayoría de los hombres realizan trabajos extra-agrícolas en diferentes ciudades o regiones de México, ya sea como obreros de la construcción o como comerciantes ambulantes, y en la última década ha presentado migración hacia los Estados Unidos de Norteamérica de forma ilegal (VIZCARRA, 2012). Por su parte, las mujeres jóvenes también se han insertado a los mercados de trabajo asalariados, casi siempre como empleadas domésticas en las ciudades, pero una vez que se casan, se convierten en cuidadoras del hogar, de la familia y de los recursos masculinos dentro de la comunidad (la parcela o milpa, la casa o algún local comercial), e inclusive las mujeres están sustituyendo a los hombres en trabajos comunitarios y políticos mientras ellos no se encuentran en la localidad (GUADARRAMA; VIZCARRA; LUTZ, 2009). 
Por otro lado, en el 90\% de los hogares las jefas de hogar son beneficiarias del Programa Oportunidades, lo que somete a las madres a prácticas de regulación gubernamental que vigila el cumplimiento de los compromisos que adquieren al recibir los apoyos monetarios directos y servicios de salud. En la última década las familias beneficiarias de este programa fueron inscritas al Seguro Popular ${ }^{7}$, obteniendo con ello servicios gratuitos (no condicionados) para la prevención y atención a la salud. A través de las relaciones que se han establecido entre instituciones gubernamentales y la población, observamos que las mujeres con mayor experiencia en programas asistenciales, por lo general también adquieren apoyos suplementarios que ofrecen otras agencias gubernamentales o no gubernamentales como lo es Un Kilo de Ayuda que consiste en despensas alimentarias mensuales.

Además de las tortillas de maíz, se puede decir que la dieta diaria de los hogares de San Jerónimo Mavatí dejó de ser "tradicional" (maíz, frijol, chile, frutos de estación, arvenses o quelites) para convertirse en una dieta "variada" (con abundancia en carbohidratos). Esto, gracias a que con los ingresos obtenidos de diferentes fuentes y a la proliferación de tiendas locales que se abastecen de productos alimentarios procesados provenientes de las empresas transnacionales con gran capacidad de distribución, las mujeres tienen acceso con mayor facilidad a estos alimentos. Esta dieta no es considerada adecuada, pese a que muchos alimentos tradicionales siguen estando en la estructura nutrimental, pues su importancia relativa ha disminuido ante la presencia de alimentos altos en azúcares, carbohidratos y lípidos. De las 1,750 calorías que en promedio se consume, las tortillas de maíz aportan sólo el $27 \%$ del total, poco más de lo que aportan las bebidas azucaradas y carbonatadas $(20 \%)$. El resto proviene de sopas de pasta e instantáneas, galletas, pan blanco, arroz, cereales de caja, leche, frutas, leguminosas, huevo, pollo y atoles, entre otros, de menor importancia dentro de la estructura. Ahora bien, los hogares mazahuas tienden a consumir sólo dos comidas al día en casa, casi siempre preparadas por las mujeres, pero ante la ausencia masculina y con sus nuevos compromisos ante la comunidad y con el gobierno, han sustituido gradualmente los alimentos tradicionales que requerían largas horas de preparación, y grandes cantidades de combustibles vegetales (leña) por aquellos industrializados que generalmente se preparan rápidamente como son las sopas de pasta e instantáneas que, acompañadas de bebidas azucaradas carbonizadas, resultan tener un alto contenido de carbohidratos refinados que afectan al estado general de la salud de la población. 
Existen varias preocupaciones externadas por la médica de la clínica local: una de ellas es que última década han aumentado los casos de Diabetes Mellitus 2, afectando cada vez más a poblaciones entre 35 y 50 ańos. Otra es que persisten los nacimientos de bebés con bajo peso, aunado a que en nuestros estudios detectamos que dos de cada diez menores de cinco ańos de edad tiene algún grado de desnutrición ${ }^{8}$ y uno de cada 10 en el mismo rango de edad presenta obesidad. Indudablemente, la leche materna es el alimento predilecto hasta los 2 años que se da el destete y la ablactación comienza a temprana edad (3 meses). Son pocas las mamás que con mayores años de escolaridad y con experiencia laboral extra-agrícola, procuran ir introduciendo alimentos blandos a la dieta de sus bebés (frutas). La mayoría de ellas comienzan esta etapa con una tortilla, pan blanco y galletas. A veces se observan golosinas en las manitas de bebés, y las bebidas endulzadas y/o carbonatadas son puestas en sus bocas (en dosis pequeñas) directamente de los envases que toman sus mamás, o hermanos(as) mayores.

De las y los 80 infantes entre 5 y 12 ańos a quienes tomamos medidas de peso y talla para sacar su índice de masa corporal (IMC) (49\% nińos, $51 \%$ niñas), el $30 \%$ presentaba desnutrición por baja talla, pero sólo $2 \%$ presenta bajo peso; el $78 \%$ tiene peso normal y $20 \%$ ya tiene obesidad. La niñas tienen mayor prevalencia por baja talla y menor en obesidad con respecto a sus pares masculinos, probablemente porque ellas desde pequeñas realizan trabajos domésticos, tarea que no acaba al pasar de los años y pese a que ellas también van a la escuela (VIZCARRA; MARÍN, 2006).

En 1996 llegó la luz eléctrica a la comunidad, y poco después las antenas de televisión comenzaron a formar parte del paisaje rural mazahua. En la actualidad, uno de cada cuatro hogares tienen televisor y ahí los nińos más que las niñas pasan tiempo mirándola después de haber cumplido con sus obligaciones domésticas y agrícolas del hogar. Al mismo tiempo que llegaron las televisiones, en las tiendas de la localidad se instalaron máquinas de videojuegos donde también los niños pasan horas jugando después de salir de la escuela.

\section{Otomíes de San Andrés Cuexcontitlán}

Perteneciente al municipio de Toluca ${ }^{9}$, San Andrés Cuexcontitlán es considerada una localidad periurbana con raíz otomí, ya que sus ancestros fueron pobladores originarios del noreste del Valle de Toluca. Para el 2010 la localidad 
alcanzó 18,005 habitantes (49.3\% hombres, 50.7\% mujeres), distribuidos en 3,802 , y donde el $23 \%$ está siendo jefaturado por mujeres (CONAPO-INEGI, 2011), a razón de casi 5 miembros por familia. El 22.2\% de la población total aún conserva la lengua otomí, siendo en su generalidad mayores de 50 ańos (SEDESOL, 2012). No obstante, se considera una comunidad relativamente joven porque la mitad de su población es menor de 35 años de edad. Uno de los problemas de esta juventud es que existe un porcentaje alarmante de deserción escolar entre jóvenes de ambos sexos. Además, el analfabetismo sigue siendo una característica de la población otomí, afectando principalmente a mujeres mayores de 30 años. Como consecuencias de estos fenómenos demográficos, han proliferado las bandas de jóvenes en la calle (algunas delictivas), y se ha registrado uno de los mayores índices de madres solteras menores de 18 ańos en el Valle de Toluca (información compilada por autoridades de salud).

En la última década, la localidad tuvo un crecimiento poblacional acelerado de 22.6\% (3,318 habitantes) (CONAPO-INEGI, 2011) debido a que se han instalado zonas residenciales para clases medias altas, teniendo implicaciones sociales para los hogares originarios. Por un lado, la expansión de la ciudad de Toluca hacia esta zona los absorbió en la mancha urbana y junto con ella se ha manifestado un proceso de aculturación apresurado a través del arribo de patrones de consumos urbanos culturales. Por otro lado, coexisten tradiciones en la organización en torno a las estrategias de reproducción social propias del pueblo Otomí que tratan de resistir a estos embates. Un hecho relevante que refleja la complejidad de la vida periurbana otomí, es la construcción de escuelas privadas y centros comerciales en la periferia de la comunidad. Esto es, $\mathrm{n} n$ el sistema educativo público de San Andrés existen 3 escuelas primarias públicas (con dos turnos), 3 pre-escolares (una con dos turnos), y una secundaria técnica (con dos turnos). Por parte del sistema privado, existe un colegio que colinda entre esta localidad y otra también de origen Otomí llamada San Pablo Autópan, que ofrece educación desde el nivel preescolar hasta el nivel preparatoria ${ }^{10}$.

La mayoría de los y las estudiantes que asisten a las escuelas públicas dicen no hablar otomí y no se piensan indígenas, y tienden a vestir de forma homogénea (jeans, tenis, camisetas con impresos etc.), a peinarse a la moda y a escuchar música pop en español e inglés. Algunos tienen teléfonos celulares y frecuentan los cibercafés que se han multiplicado en la última década. Pero también continúan conviviendo con sus sistemas de creencias otomíes cívicoreligiosas mediante el consumo de tortillas, escuchando música de banda, y 
conservando su baño de temazcal en sus hogares. Un dato sobresaliente es que el $94 \%$ de la población infantil que participó en nuestro estudio afirmó tener al menos un pariente de primera línea (por lo general abuelas y abuelos) que habla la lengua indígena otomí (CEBALLOS, 2012b). En términos de García Canclini (1995), lo que se aprecia en esta localidad periurbana es una hibridación cultural, cuyos efectos modernizadores de la urbanización al estilo estadounidense (residencias periféricas) y de la expansión de consumos globales a través de los medios masivos de comunicación, no han eliminado las formas culturales tradicionales, sean éstas indígenas o populares, sino más bien pretenden sobrevivir con sus tradiciones en el proceso modernizador en la era de la globalización. Un ejemplo de este proceso es la alimentación. Seguro, no se puede negar que la agricultura basada en el cultivo de maíz ha dejado de ser la principal actividad de la comunidad, y a pesar de la persistencia de parcelas manejadas a una altitud de 2,590 m.s.n.m., se observa el fenómeno de la residencialización, las tortillas de maíz siguen siendo parte de la dieta diaria de los hogares, aunque muchas de ellas se compran en tortillerías que utilizan harina de maíz industrializado. De esta manera, el consumo de maíz corresponde al $20 \%$ del total del aporte calórico de la dieta promedio de los habitantes de San Andrés. El resto lo ocupan las bebidas azucaradas y/o carbonizadas, las sopas de pasta e instantáneas, las frituras de harina de trigo, pan, galletas, aceites, leguminosas, pollo, huevo y golosinas (CEBALLOS, 2012a).

$\mathrm{Al}$ igual que la comunidad mazahua, sin excepción, todos los hogares originarios realizan actividades extra-agrícolas para ganarse la vida (obreros de la construcción, comerciantes, choferes, servicio doméstico a domicilio etc.). Por sus bajos ingresos son categorizados como pobres, siendo que más de 1900 hogares están inscritos al programa Oportunidades y al Seguro Popular. Entre menor es el ingreso más lo dedican al gasto de alimentos, de tal forma que se observa un patrón de dieta alimentaria parecida a los hogares mazahuas, la cual se refuerza con presencia de autoconsumo "rural". No obstante, en términos de consumo calórico, entre el mayor es el ingreso mayor se consumen calorías basadas en carbohidratos. Por ejemplo, los hogares con menores ingresos consumían en promedio por persona/día 1,700 calorías y con mayores ingresos, 3,200 calorías (CEBALLOS, 2012a). En este conteo, no se tomaron en cuenta el consumo de bebidas alcohólicas, sin embargo los directivos de las escuelas externaron que uno de los problemas más graves de la comunidad son las adicciones a estas bebidas $\mathrm{y}$ algunas drogas que ya se consiguen fuera de las escuelas. 
En comparación con los hogares mazahuas, los padres no están ausentes porque trabajan en el valle de Toluca. Ellos comen todos los días en el hogar a su regreso por las tardes noches, pero también un gran número de mujeres se han insertado como fuerza laboral remunerada fuera de la localidad, lo que las ha sometido a dobles jornadas de trabajo, reduciéndoles considerablemente el tiempo para preparar alimentos en casa y obligándolas a comprar alimentos procesados baratos, e inclusive a los niños y las niñas se les da dinero para que compren alimentos en la escuela o fuera de casa. Por el monto con el que disponen, esta población compra alimentos baratos y pobres en nutrimentos de calidad pero ricos en azúcares refinados (golosinas), almidones y harinas, grasas saturadas, chiles, y sales, lo que vulgarmente se le ha llamado "comida chatarra", la que a al mismo tiempo que satisface el apetito infantil agrada al paladar.

Las consecuencias de la hibridación alimentaria se reflejan directamente en el estado nutricional de su población infantil. Ceballos (2012b) reportaron que el $73.8 \%$ de niños y niñas de preescolar ( 4 a 6 años) tienen peso normal para su edad según el sexo, sin embargo casi 3 de cada 10 infantes ya presentan obesidad. Prácticamente la desnutrición infantil está desapareciendo en la comunidad con un índice de entre 1.3 y 1.6 para edades desde 4 hasta 15 años). En lo que respecta a la edad escolar de 6 a 12 años, el peso normal en promedio para ambos sexos es de $64.48 \%$, aumentando la prevalencia de obesidad, principalmente en los niños (36.35) con respecto a sus pares niñas (23.72). Por su parte, a nivel de escolares de secundaria entre 12 y 16 años de edad, 4 de cada 10 mujeres adolescentes presenta obesidad, y alrededor de 2 de cada 10 hombres jóvenes tiene obesidad. Vale la pena enfatizar que en general la niñas tienen mayor porcentaje de sobrepeso y los niños de obesidad para todos los rangos de edad que van desde los 4 hasta los 16 años, a pesar de que estas cifras están ligeramente por debajo de las medias nacionales registradas por la Encuesta Nacional de Salud y Nutrición (INSP-ENSANUT, 2012), debido a la condición de pobreza que vive esta población, es meritorio tener atención especial por parte de las instituciones de educación y salud.

Otro dato relevante de nuestro estudio, fue que a estudiantes de la escuela secundaria se les preguntó si tenían algún pariente cercano con Diabetes y la respuesta fue sorprendente; el 88\% de más de 1,200 estudiantes contestaron que sí. Ello refleja sin lugar a duda una preocupación para el sistema de salud pública del municipio y del Estado. 


\section{Resignificación de identidades de la niñez indígena con obesidad}

La dieta campesina e indígena mexicana se consideró discursivamente y por mucho tiempo como comida de pobres, poco variada, escasa o deficitaria en proteínas, y se relacionó directamente con la inseguridad alimentaria y ésta con la desnutrición y sus elevados índices de morbilidad y mortandad infantil. Estos discursos penetraron en las formas de estigmatizar a las poblaciones indígenas y pobres de México, siendo motivo de discriminaciones de clase y étnicas (VIZCARRA, 2002; 2008). Tal vez por eso, las madres de los y las menores de 11 ańos de las dos comunidades de estudio, no perciben la obesidad como un problema de salud. Al contrario, mencionaron que los gorditos y las gorditas son sinónimo de niños y niñas bien alimentados(as) y por lo tanto saludables, e incluso lo relacionan con el estatus social del hogar (menos pobres). De la misma manera relacionaron el bajo peso con enfermedad y pobreza, mostrando aflicción por ese estado. Vale la pena mencionar que la mayoría de las madres que percibían estas relaciones no tenían la educación primaria terminada o no saben leer ni escribir.

Por otro lado, las madres de adolescentes mujeres entre 11 y 15 años, que presentaron obesidad se mostraron preocupadas por sus hijas y no así por sus hijos. Es decir que la obesidad masculina en esa edad no es considerada un motivo de alarma para sus madres. Si bien, la percepción materna de la obesidad para ambos géneros no está asociada con la enfermedad o con un factor de riesgo, la obesidad de las jóvenes mujeres resulta ser motivo de crítica y discriminación entre el mismo grupo de edad, de aquí que las madres de ambas comunidades se mostraron preocupadas. En tanto que las madres mazahuas y otomíes con mayores niveles de educación (en años estudiados) se percibieron como obesas a pesar de que desconocían su IMC, manifestaron estar molestas con la estética de su cuerpo, y en repetidas ocasiones hicieron referencia a que desearían ser delgadas tal y como lo eran antes de tener hijos e hijas. Y en efecto, una vez que se tomaron sus medida más de la mitad de ellas tenían obesidad, y por las circunferencias de sus cinturas, se estimó que este tipo de obesidad ginecoide es común entre mujeres que han tenido varios embarazos sin reposos espaciados entre ellos, y a pesar de que no está directamente asociada con tener hígado graso, no se descarta que pueda ser considerada como un factor de alto riesgo para desarrollar diabetes (BAUDRAND; ARTEAGA; MORENO, 
2010), aunque lo que más inquieta a estas mujeres no es la salud sino la estética de su cuerpo. Las mujeres que trabajan en la ciudad de Toluca por ejemplo, externaron la inquietud de adquirir productos para bajar de peso, y en ambas comunidades las mujeres con más años de educación ya estaban familiarizadas con el conocimiento de dietas y rutinas de ejercicios para adelgazar.

Estas manifestaciones de sí mismas son prologadas hacia sus hijas, teniendo mayor efecto en la comunidad Otomí, debido al acercamiento continuo con los referentes urbanos occidentalizados sobre las modelos de tallas cero. Tal es la influencia que en la escuela secundaría se presentaron dos casos de bulimia y anorexia en niñas de 12 y 13 años. Esto resulta contradictorio cuando las madres y abuelas no han cambiado el orden social de cómo y qué se le asigna la identidad de género a sus hijos e hijas. Es decir, a ellas se les asignan roles que tienen que ver con el cuidado y servicio de los demás como la maternidad, a través de obligarlas a realizar actividades domésticas desde tempranas edades y hacerse cargo de sus hermanito(as) más pequeños(as). Al mismo tiempo que se ven obligadas a asistir a la escuela como compromiso adquirido por el programa Oportunidades, ellas comienzan a tener aspiraciones diferentes a las de sus madres, como tener una carrera profesional, tener menos hijos, $\mathrm{y}$ a vestirse mejor y obtener cuerpos delgados. A ellos se les asigna la fortaleza y destreza para salir adelante como proveedores. Los niños mazahuas sólo piensan en terminar la secundaria para ir a trabajar fuera de la comunidad y de ser posible emigrar hacia los Estados Unidos de Norteamérica. Por su parte, los niños otomíes son más rebeldes con sus madres, imitando actitudes "machistas" que reproducen de sus padres quienes por lo general no participan en las labores domésticas pese a que sus madres trabajan fuera del hogar. Esta situación ha propiciado un incremento en la violencia doméstica relacionada casi siempre con el exceso de consumo de bebidas alcohólicas por parte de sus padres y hermanos mayores. En cuanto a sus cuerpos, la obesidad no es importante sino hasta la edad de 14 años, pues la mayoría referían a sus padres como "barrigones" pero fuertes.

\section{En la escuela}

A finales de enero de 2009, la Secretaría de Salud puso en marcha el programa "5 Pasos por su Salud, para Vivir Mejor", que promueve hacer ejercicio, beber agua, comer sanamente, medirse y comparar su peso en diferentes días, con el objetivo de combatir el sobrepeso y la obesidad en el 
país. Un año después este programa se puso en práctica en las escuelas de educación básica (preescolar, primaria y secundaria) en todo el país a través de la Secretaría de Educación Pública. La idea era poner en práctica las estrategias para evitar el sobrepeso y la obesidad en los preescolares y escolares a través del fomento de estilos de vida saludables. En las escuelas de la comunidad Otomí este programa llegó ese mismo año, en cambio en la comunidad mazahua llegó un año después, en septiembre de 2011. Hasta antes de esas fechas respectivas, en las escuelas de educación básica no existía el problema de la obesidad infantil. Aún así comenzaron a implementar estrategias de activación física, que consistía en media hora al día y, daban pláticas a las madres sobre los beneficios del "plato de buen comer", el cual se muestra gráficamente un plato dividido en fracciones relativas, sobre las recomendaciones de consumo diario para cada grupo de alimentos, sin tomar en cuenta los modos de vida locales y sus dinámicas cambiantes, ni tampoco se consideró que en las comunidades como la mazahua no existe acceso directo al agua potable, por lo contrario, éste es un recurso hídrico que escasea en época de secas. Fue hasta entonces que los niños y nińas que aparentemente tenían mayor peso corporal que el resto de la comunidad escolar comenzaron a ser señalados de forma discriminatoria por sus mismos(as) compañeros(as) como gordos, gordas, obesos u obesas. De hecho en la primaria de la comunidad otomí, el personal docente refirió que estudiantes practican "Bullying" o acoso escolar a los o las que son más gorditos(as) en la escuela. Problema que no existía antes en la escuela, al menos por esa razón.

Esta nueva discriminación se vio reflejada cuando estábamos tomando las mediadas para obtener el IMC de escolares en ambas comunidades. Sobre todo las nińas que se sentían con problemas de obesidad pedían que no estuviera presente ninguna compañera en el momento que ella estaba siendo medida para que nos supieran sus datos. Tanto niños como nińas, pedían consejos no solo para evitar la obesidad, sino para bajar de peso. Ellas deseaban tener cuerpos delgados como las artistas juveniles; ellos preferían consejos para ser deportistas $\mathrm{o}$ atletas musculosos y altos. Indudablemente se referían a estereotipos que no corresponde a las estructuras antropométricas mexicanas y mucho menos a las de sus raíces indígenas.

Otra de las medidas que se trató de implementar en las escuelas a nivel nacional fue la regulación de la venta de alimentos no aptos para el desarrollo y crecimiento saludable de la niñez en México. El debate nacional alcanzó todas 
las esferas políticas, económicas y sociales, pero finalmente se impuso la lógica neoliberal y capitalista, exigiendo la no censura de la venta de alimentos y bebidas procesados en las cadenas agroalimentarias transnacionales y nacionales, y más bien promoviendo junto con el gobierno, transferir la responsabilidad del bienestar infantil a las madres y los padres de familia, así como a los docentes, para que en conjunto se eduquen a los niños y las niñas para elegir alimentos sanos en los espacios de recreación escolar. Como resultado, observamos que aún con la voluntad de toda la comunidad, la propuesta era complicada ya que por ejemplo, mientras que el costo de una manzana que aporta 57 calorías aproximadamente era de 5 pesos (medio dólar), una bolsa de chicharrones (fritura elaborada con harina de trigo y guisadas en aceite vegetal o grasa animal) con salsa picante y limón costaba lo mismo, pero su aporte es de alrededor de 250 calorías. Esto resultaba conveniente en términos de aporte energético, pues muchos niños y niñas llegaban a la escuela sin desayunar y sin un almuerzo preparado en casa para comerlo como lunch en la escuela.

No menos importante, el personal docente es otra figura que interviene en la percepción que los niños y las niñas tienen de la alimentación y de la obesidad. De forma contradictoria, el personal docente que promueve estilos de vida saludables en la escuela, en su mayoría presentan obesidad, algunos tienen diabetes, y comen los alimentos que condenan en sus enseńanzas y delante de sus estudiantes. Estas actitudes desvalorizan los contenidos de los programas y generan confusión entre los y las infantes, por tratarse de patrones de conducta alimentaria "ocultas" (PIAGGIO, 2011).

Por todo lo anterior, se puede decir que la redefinición de las identidades infantiles de mazahuas y otomíes es compleja, porque interviene un proceso de intersubjetivación entre lo idóneo ajeno a su cultura, entre lo contradictorio de lo que se dice y no se hace, y entre lo disponible que se legitima en la conciencia colectiva sobre el sentido de pertenencia local según su edad y su género.

\section{Reflexiones finales}

La influencia de las dietas de adelgazamiento difundidas por diversos medios de comunicación y mercados se ven reflejadas en las preocupaciones por la obesidad, siendo más una cuestión estética y de aceptación que un asunto de salud. Ser obeso y, sobre todo, obesa, constituye en nuestros días un auténtico estigma social. La presión contra este padecimiento es muy 
clara: en el momento actual el atractivo físico (sobre todo en las mujeres), sí se centra en el cuerpo, aunque no dependa sólo de su volumen (FISCHLER, 1995). Al igual que otros estudios que han señalado que las creencias y valores, nivel educativo materno, sexo del hijo, desconocimiento de una definición del sobrepeso y obesidad o el hecho de no aceptar que el hijo presenta estas patologías pueden ser factores que influyen en la percepción maternal no adecuada del sobrepeso y obesidad de sus hijos e hijas (PÉREZ GIL, VEGA; ROMERO, 2007). Esto tiene implicaciones importantes si se considera que la percepción es un proceso propio de la persona, influido por la relación que se tiene con el sujeto percibido, las experiencias y las creencias asociadas con un fenómeno específico.

Este trabajo destacó la necesidad de considerar que el riesgo de desarrollar sobrepeso u obesidad no es exclusivo de la persona con origen indígena que se mueve entre lo rural y lo urbano. Con la llegada masiva de productos asociados con la globalización alimentaria, y los ideales de cuerpos occidentalizados (delgados, deportivos y estilizados), se perciben cambios importantes tanto en los regímenes alimentarios de los pueblos como en la percepción de sus cuerpos. Si antes un factor limitante para acceder a alimentos saludables era el ingreso, se sabe que aún con éste, frente a la nueva accesibilidad de nuevos alimentos procesados, sigue practicándose una mala distribución del ingreso dentro del hogar, y por tanto el estado de nutrición y salud de sus integrantes es afectado de manera diferenciada (edad, género). En efecto, después del género, los procesos de diferenciación social por pertenecer a un grupo étnico, por su marginalización y pobreza son marcadores definitivos de discriminación en la niñez, aunado a los marcadores que se inscriben en sus cuerpos estigmatizados por su forma y no por su salud, constituyen las nuevas generaciones en un horizonte de exclusión social que no registra esas diferencias.

Un hecho que necesita ser subrayado es que la obesidad de la pobreza no existía en las comunidades indígenas antes de que el Estado resolviera la seguridad alimentaria con la dotación de ingresos para comprar los alimentos que no pueden producir. Específicamente se debe tener una reflexión crítica sobre las consecuencias que ha tenido el programa Oportunidades en estas transiciones nutricionales, pues después de 15 años de existir (incluyendo al PROGRESA), los pobres no han dejado de ser pobres, y los problemas de salud pública que aquejaban a estas poblaciones han pasado de enfermedades 
infecciosa a crónicas degenerativas. Asimismo, debemos retomar las falsas visibilidades de las infancias plenas de derechos dentro de los programas de prevención de la obesidad e inclusive de combate al hambre ya que se tiende a homogenizar los problemas y se construyen necesidades de intervención para solucionar el problema a través de responsabilizar a las madres y a los infantes mismos por su obesidad. Pero al salir del discurso sobre el ideal de la vida saludable, el contexto donde interactúan sigue sin cambiar. Es decir, saturación de publicidad y productos para consumir alimentos no aptos para ese ideal occidentalizado de vida saludable, por ejemplo.

La actividad física continua, buenos hábitos dietéticos, conciencia de la salud-enfermedad, conocimiento de lo que es el sobrepeso y la obesidad, entre otras prácticas contempladas en "paquetes" de salud integral, si bien son factores determinantes e intervienen para lograr el máximo desarrollo potencial infantil (SOLANO; BARON; REAL, 2005), estos pueden verse limitados ante el desconocimiento de los usos, costumbres e hibridaciones de las culturas locales, sobretodo, de aquellas que están en un continuo proceso de transformación por su interacción con el crecimiento urbano y los consumos globales. En este sentido, la percepción que un(a) infante tiene de sí mismo(a) a través de su imagen corporal, no sólo depende de una construcción biográfica o identitaria, sino que ésta está atraviesa por una serie de intervenciones discursivas y subjetivas, que ni el contexto local, ni la cultura étnica, pueden resistir a reproducirlas, generando con ello actitudes de autovaloración, cuyos límites de aceptación obedecen no sólo a intervenciones institucionales (CARBALLO; CRESPO, 2003), sino sobretodo a la formación de una sociedad crítica que promueva un cambio social consciente.

Por último, enfatizamos en la necesidad de continuar trabajando en esta temática, abordándola desde una metodología cuantitativa y cualitativa para comprender mejor el tema y tener más elementos que contribuyan de alguna manera a la formulación de políticas alimentarias y nutricionales que rescaten los aspectos culturales simbólicos sobre el cuerpo y la transición alimentaria. Tal vez así estaremos dando un gran paso en la comprensión de la complejidad que rodea la redefinición de las identidades infantiles indígenas entre comer, alimentarse, dejar de ser pobre y su relación con el cuerpo, tanto como un organismo sujeto a un proceso de salud-enfermedad, como en la construcción simbólica de las identidades que las mujeres y los hombres hacen de él. 


\section{Notas}

${ }^{1}$ En este escrito la obesidad refiere tanto al sobrepeso como a la obesidad propiamente dicha. De manera general, la obesidad y el sobrepeso se definen como una acumulación anormal o excesiva de grasa que supera el $20 \%$ de la masa corporal lo que resulta ser un problema para la salud. Dependiendo de una medición simple de peso, talla, edad y sexo, se puede diferenciar si un niño tiene sobrepeso u obesidad. Sin embargo estudios pediátricos han alertado que una vez que se presenta el sobrepeso en la infancia, existen altas probabilidades de que pasen a tener obesidad en la adolescencia y prolongarse en la edad adulta de no intervenir para evitarlo (COLE, 2004).

${ }^{2}$ El programa Oportunidades forma parte de las políticas sociales para combatir la pobreza extrema en México. Su antecesor es el Programa de Educación, Salud y Alimentación (PROGRESA) de 1998 a 2002, con una metodología de focalización, se le transfieren a las madres que tienen hijos en edad escolar y menores de cinco años. Se trata de transferencias monetarias directas, condicionadas a cumplir una serie de requisitos que se integran en el bienestar salud, asistencia escolar de sus hijos, salud preventiva e higiene.

${ }^{3}$ Como ejemplo véase El Universal en: <http://www.eluniversal.com.mx/ notas/547368.html>. Consultado el 19 mar. 2013.

${ }^{4} \mathrm{El}$ proyecto inició en octubre de 2011 y tendrá una duración de 4 años. Es financiado por el Consejo Nacional de Ciencia y Tecnología en México.

${ }^{5}$ Este proyecto concluyó en enero de 2012 y fue financiado por la Universidad Autónoma del Estado de México".

${ }^{6}$ En la percepción social intervienen las características y la información almacenada sobre la persona observada e información sobre el contexto. A su vez, esta información es afectada por las características del sujeto que percibe o estable la relación con su entorno, tales como la edad y la escolaridad, lo que genera respuestas o juicios acerca de esa realidad y de la persona observada (SALAZAR, 2003).

${ }^{7}$ En 2002 nació la propuesta de ofrecer asistencia de salud básica a quienes carecieran de asistencia social, surgiendo para este propósito la necesidad 
de un "Seguro Popular de Salud". Actualmente el 95\% de esta población que carecía de servicios de salud ya tiene acceso a ellos a través de este seguro que además es gratuito.

${ }^{8}$ Existen tres clasificaciones de desnutrición para menores de 5 años: bajo peso (peso por edad por sexo), baja talla (talla por edad por sexo) y emaciación (peso/talla por edad por sexo).

${ }^{9}$ La ciudad de Toluca (municipio del mismo nombre) es la capital del Estado de México.

${ }^{10}$ En México el sistema educativo está dividido en tres grandes niveles: El primer nivel "Básico" está subdividido en tres, dado su obligatoriedad constitucional: el preescolar el cual se cursa en dos o tres grados ( 3 a 6 años de edad), la primaria con seis grados (6 a 12 años de edad) y la secundaria con tres grados (12 a 15 años de edad); el segundo nivel refiere a la educación Media Superior con tres grados de preparatoria o bachillerato; y el tercer nivel es la educación de nivel Superior (Universidad).

\section{REFERÊNCIAS}

AMIGO, Hugo. Obesidad en el niño en América Latina: situación, criterios de diagnóstico y desafíos. Cadernos de Saúde Pública, Rio de Janeiro, n. 19, p.1-13, 2003.

ARIĖS, Philip. Centuries of childhood: a social history of family life. New York: Vintage Books, 1962.

BERGER, Peter; LUCKMANN, Thomas. La construcción social de la realidad. Buenos Aires: Amorrortu editores, 2006.

BAUDRAND, René; ARTEAGA, Eugenio; MORENO, Manuel. El tejido graso como modulador endocrino: cambios hormonales asociados a la obesidad. Revista Médica de Chile, Santiago, v. 138, n. 10, p. 1294-1301, oct. 2010. Disponible en: <http://www.scielo.cl/scielo.php?script=sci_arttext \&pid=S003498872010001100015\&lng=es $>$. Acceso en: 20 abr. 2012.

BOISIER, Sergio. ¿Hay espacio para el desarrollo local en la globalización? Revista de la CEPAL Santiago de Chile, n. 86, p. 42-68, 2005. 
CALZADA LEÓN, Raúl. De desnutrición a obesidad. Acta Pediátrica, Ciudad de México, v. 24, n.1, p. 1-3, 2003.

CARBALLO, Carlo; CRESPO, Bettina. Aproximaciones al concepto de cuerpo. Perspectiva, Florianópolis, v. 21, n. 1, p. 224-227, jan./jun. 2003.

CARLI, Sandra (Org.). La cuestión de la infancia: entre la escuela, la calle y el shopping. Buenos Aires: Paidos, 2006.

CARMONA, Maricela; VIZCARRA, Ivonne. Obesidad en escolares de comunidades rurales con alta migración internacional en el México central. Población y salud en Mesoamérica, San José, v. 6, n. 2, ene./jun. 2009. Disponible en: <http://www.redalyc.org/articulo.oa?id=44660209>.

CEBALLOS, Carmen Liliana. Inseguridad alimentaria en términos de bienestar social y presencia de obesidad en más de un miembro de los hogares de origen Otomi de una localidad de Toluca. 2012. Tesis (Maestría en Ciencias de la Salud) - Universidad Autónoma del Estado de México, Toluca, 2012a.

CEBALLOS, Carmen Liliana et al. Sobrepeso y obesidad en preescolares y escolares de una comunidad periurbana de origen otomí del Valle de Toluca, México. Población y salud en Mesoamérica, San José, v. 10, n. 1, jul./dic. 2012b. Disponible en: <http://www.redalyc.org/articulo. oa?id $=44623233002>$.

COCKBURN, Tom. Children and the feminist ethic of care. Childhood, New York, v.12, n. 1, feb. 2005.

COLE, T. J. Children grow and horses race: is the adiposity rebound a critical period for later obesity? BioMed Central Pediatrics, Bethesda, v. 4, n. 6, 2004. Disponible en: <http://www.ncbi.nlm.nih.gov/pmc/articles/ PMC394330/>. Acceso en: 29 nov. 2010.

CONAPO-INEGI. Consejo nacional de Población - Instituto Nacional de Estadística, Geografía e Informática. Censo de Población y Vivienda 2010: principales resultados por localidad (ITER). México: INEGI, 2011.

FISCHLER, Claude. Gastro-nomía y gastro-anomía: sabiduría del cuerpo y crisis biocultural de la alimentación contemporánea. In: CONTRERAS, Jesús (Org.) Alimentación y cultura: necesidades gustos y costumbres. Barcelona: Universidad de Barcelona, 1995. p. 357-380. 

escolar en México..

FRIEDMAN, Jonathan. Cultural identity \& global process. London: Sage Publications, 1995.

FRONES, Ivar. Changing childhood. Childhood, New York, v.1, n.1. 1990.

GARCÍA CANCLINI, Néstor. Consumidores y ciudadanos: conflictos multiculturales de la globalización. México: Grijalbo. 1995.

GREENHALGH, Susan. Controlling births and bodies in Village China. American Ethnologist, v. 21, n. 1, p.3-30, Arlington, VA, 1994.

GOTTLIEB, Alma. The afterlife is where we come from: the culture of infancy in west Africa. Chicago: The University of Chicago Press, 2004.

GOTTHELF, S.; ARAMAYO, M.; ALDERETE, S. Coexistencia de obesidad $y$ desnutrición en familias pobres. 2004. Monografia (Postgrado a distancia en obesidad) - Universidad de Favaloro, Buenos Aires, 2004. Disponible en: <http:// www. nutrinfo.com/ pagina/info/ob04- 04.pdf>. Acceso en: 29 mayo 2008.

GUADARRAMA, Xóchitl, VIZCARRA, Ivonne; LUTZ, Bruno. De la migración: ausencias masculinas, reacciones femeninas mazahuas. Revista Relaciones del Colegio de Michoacán, Michoacán, v. 30, n.118, p. 183-219, 2009.

HAMILTON, Annette. Nature and nurture: aboriginal child-rearing in north-central Arnhem Land. Canberra: Australian Institute of Aboriginal Studies, 1981.

HARKNESS, Sara; SUPER, Charles. The cultural construction of child development: a framework for the socialization of affect. Ethos, Michigan, v.11, p. 221-223, winter 1983. Disponible en: <http://www.jstor.org. libproxy.albany.edu/stable/pdfplus/640206.pdf?acceptTC=true>. Acceso en: 22 mar. 2013.

IMSS. Instituto Mexicano del Seguro Social. Programas Integrados de Salud. 2010. Disponible en: <http://www.imss.gob.mx/programas/prevenimss>. Acceso en: 20 mar. 2013. 
INSP-ENSANUT. Encuesta Nacional de Salud y Nutrición 2012: resultados nacionales. Cuernevaca: Instituto Nacional de Salud Pública, 2012. Disponible en: <http://ensanut.insp.mx/informes/ ENSANUT2012ResultadosNacionales.pdf>. Acceso en: 18 mar. 2013.

ISUNZA, Vera A. La obesidad en la pobreza: violación al derecho a la alimentación. 54f. 2004. Monografia (Postgrado a distancia en obesidad) Universidad de Favaloro, Buenos Aires, 2004. Disponible en: <http://www. nutrinfo.com/biblioteca/monografias/ob04-02.pdfs.

LOBSTEIN, Tim; BAUR, Louise; UAUY, R. Obesity in children and young people: a crisis in public health. Obesity Research, v. 5, suppl. 1, p. 4-85, 2004.

MCLUHAN, Marshall; POWERS, Bruce E. La aldea global. Barcelona: Gedisa, 1993.

MEAD, Margaret. Coming of age in Samoa: a psychological study of primitive youth for western civilization. New York: Harper Collins Publishers, 2001.

MELÉNDEZ TORRES, Juana María; CAÑEZ DE LA FUENTE, Gloria María; FRÍAS JARAMILLO, Hevilat. Comportamiento alimentario durante la adolescencia ¿Nueva relación con la alimentación y el cuerpo? Revista Mexicana de Investigación en Psicología, v. 4, p. 99-111, 2012. (Número monográfico).

MOFFAT, Tina. The "childhood obesity epidemic": health crisis or social construction? Medical Anthropology Quarterly, Hoboken, NJ, v. 24, n. 1, p. 01-21, 2010.

PÉREZ GIL, Sara Elena; VEGA, Luz Amaranta; ROMERO, Gabriela. Alimentación de mujeres en una zona rural: ¿Existe una nueva percepción del cuerpo? Salud Pública de México, Ciudad de México, v. 49, n. 1, p. 52-62, 2007.

PEÑA, Manuel; BACALLAO, Jorge. La obesidad en la pobreza: un problema emergente en las Américas. In: PENAA, Manuel; BACALLAO, Jorge (Org.). La obesidad en la Pobreza. Washington: Organización Panamericana de la Salud, 2000. p. 3-11. 
PIAGGIO, Laura; CONCILIO, Celeste; ROLON, Marina; DUPRAZ, Silvina. Alimentación infantil en el ámbito escolar: entre patios, aulas y comedores. Salud colectiva, Buenos Aires, v. 7, n. 2, p. 199-213, mayo/ago. 2011. Disponible en: <http://www.scielo.org.ar/scielo.php?pid=S185182652011000200012\&script=sci_arttext>. Acceso en: 12 ene. 2012.

POPKIN, Barry M. The nutrition transition and obesity in the world. Journal of Nutrition, Bethesda, MA, n. 131, p. 871S-873S, 2001.

PROUT, Alan; JAMES, Alison (Org.). A new paradigm for the sociology of childhood? Provenance, promise and problems. In: PROUT, Alan; JAMES, Alison. Constructing and reconstructing childhood. Londres: Falmer Press, 1990. p. 7-35,

SALAZAR, J. M. et al. Percepción social. Asociación Venezolana de Psicología. Ciudad de México: Trillas, 2003. p. 77-108.

SARGENT, Caroline; HARRIS, Michael. Gender ideology, childrearing, and child health in Jamaica. American Ethnologist, Hoboken, NJ, v. 19, n. 3, p. 523-537, 1992.

SASSI, Franco. Obesity and economic of prevention: fit not fat. $O C D E$ : better policies for better lives, Paris, 23 sep. 2010. Disponible en: < www. oecd.org/health/fitnotfat>. Acceso en: 17 oct. 2011.

SEDESOL. Secretaría de Desarrollo Social. Catálogo de localidades 2012. México: INEGI, Disponible en: < http://www.microrregiones.gob.mx/ catloc/>. Acceso en: 5 ene. 2013.

SOLANO, Liseti; BARÓN, María Adela; REAL, Sara del. Situación nutricional de preescolares, escolares, y adolescentes de Valencia, Carabobo, Venezuela. Anales Venezolanos de Nutrición, Caracas, v. 18, n. 1, p.72-76, 2005.

STEPHENS, Sharon (Ed.). Children and the Politics of Culture. Princeton: Princeton University Press, 1995.

URIBE, José Fernando. Las prácticas alimentarias relacionadas con la búsqueda del ideal corporal: el caso de la ciudad de Medellín, Colombia. Boletin de Antropología Universidad de Antioquia, Medellín, v. 20, n. 37, p. 227-250, 2006. 
VILLALOBOS, José Ángel Córdova et al. Estrategia 5 pasos para la salud escolar. Ciudad de México: Secretaría de Educación Pública, 2010. Disponible en: <http://sep.gob.mx/work/appsite/basica/estrategia_5_pasos. pdf $>$. Acceso en: 20 mar. 2013.

VIZCARRA, Ivonne. Entre el taco mazahua y el mundo: la comida de las relaciones de poder, resistencia e identidades. Ciudad de México: Instituto Mexiquense de la Mujer; Universidad Autónoma del Estado de México, 2002.

VIZCARRA, Ivonne. Entre las desigualdades de género: un lugar para las mujeres pobres en la seguridad alimentaria y el combate al hambre. Argumentos, Coyoacán, v. 21, n. 57, p. 141-170, may./ago. 2008.

VIZCARRA, Ivonne. Inseguridad social y alimentaria: praxis de la violencia estructural. Obesidad y Diabetes en poblaciones rurales del estado de México. In: HERNÁNDEZ, Maria del Carmen; MELÉNDEZ, Juana Maria (Org.). Alimentación contemporánea: un paradigma en crisis y respuestas alternativas. Hermosillo: Centro de Investigación en Alimentos y Desarrollo, 2012. p.105-132.

VIZCARRA, Ivonne (Org.). La feminización del campo mexicano en el Siglo XXI: localismos, transnacionalismos y protagonismos. México: Plaza y Valdés, 2013.

VIZCARRA, Ivonne; LUTZ, Bruno. Globalisation et crises alimentaires: remesas, sécurité alimentaire et pauvreté dan l'État de Mexico (centre du Mexique). In: LABRECQUE, Marie France; BOULIANNE, Manon; DOYON, Sandrinne. Migration, environnement, violence et mouvements sociaux au Mexique: dynamiques regionales en contexte d'économie globalisée. Quebec: Press de la Universidad Laval, 2010. p. 57-97.

VIZCARRA, Ivonne; MARÍN, Nadia. Las niñas a la casa y los niños a la milpa: la construcción social de la infancia mazahua. Convergencia, Ciudade de Mexico, v. 13, n. 40, p. 39-67, ene./abr. 2006.

WALLACE, Edwin R. Freud and anthropology: a history and reappraisal. New York: International Universities Press, 1983. 

escolar en México...

WHO. World Health Organization. Obesity preventing and managing the global epidemic. Génova: Report of a WHO Consultation on Obesity, 1998.

WHO. World Health Organization. World health statistics 2012.Génova, 2012. Disponible en: <http://www.who.int/gho/publications/world_health_ statistics/2012/en/index.html>. Acceso en: 21 dic. 2012.

WRIGHT, Charlotte M. et al. Implications of childhood obesity for adult health: findings from thousand families cohort study. British Medical Journal, London, v. 323, p. 1280-1284, 2001. 


\section{A obesidade na ressignificação de identidades infantis indígenas de crianças em idade escolar no México: o caso dos povos Mazahua e Otomí}

\section{Resumo}

No México, a obesidade infantil é um grave problema de saúde pública relacionado com as consequências da globalização e dos mercados agroalimentares. Esta situação atingiu as crianças otomís e mazahuas que vivem uma constante reformulaçáo de seus contextos étnicos, locais e familiares intrinsecamente interligados com os processos de globalização, que trazem como resultado a desigualdade social. Para compreender como as identidades indígenas das crianças em idade escolar foram reformuladas, este artigo apresenta um estudo sobre os contextos em que elas vivem seu cotidiano, juntamente com as variáveis de gênero, etnia, pobreza e corpo. Empregou-se uma metodologia qualitativa com dados estatísticodescritivos obtidos em duas comunidades indígenas: Mazahua e Otomí. Conclui-se que tanto o gênero como o pertencimento a uma etnia e a situação de pobreza faz com que discrimine esta populaçáo infantil e que seja estigmatizada. Assim, é incorreto responsabilizar somente as mães e as próprias crianças pelas condiçóes nutricionais em que vivem, já que suas decisóes em relação à alimentação são determinadas por instituiçóes

\section{Obesity in the redefinition of identities among the school- age indigenous children in Mexico: The case of Mazahua and Otomi people}

\section{Abstract}

In Mexico, childhood obesity is a highly public health problem related to the consequences of globalization and the agri-food market. This situation has reached Mazahua and Otomí indigenous children who are experiencing a constant reformulation of their ethnic, local and family contexts intrinsically intertconnected with the globalization process that bring social inequality as a result. In order to understand how have indigenous children's identities been reformulated, this article presents a study about the contexts in which children experience their daily life along with the gender, ethnicity, poverty and body variables. A qualitative methodology was used with additional statistical data gathered in two indigenous communities: Mazahua and Otoni. The conclusion is that gender as well as the feeling of belonging to an ethnic community and to a situation of poverty causes discrimination and stigmatization toward the indigenous children. Thus, it is incorrect to give complete responsibility to mothers and the children themselves about their nutritional conditions, since their decisions in relation to food are determined by the government, by national and international non-profit 

escolar en México..

governamentais e não governamentais nacionais e internacionais, assim como por empresas de alimentos nacionais e globais.

Palavras-chave: Obesidade. Infância. Identidade. organizations, as well as by global and national food companies.

Keywords: Obesity. Childhood. Identity.

\section{Ivonne Vizcarra-Bordi}

E-mail: ivbordi@hotamaul.com

\section{Nadia Marín-Guadarrama}

E-mail:kimi_nadiaxxi@yahoo.com

Recebido em: 31/3/2013

Versão final recebida em: $12 / 8 / 2013$

Aprovado em: 14/8/2013 